\title{
OEG Publication
}

Gómez-Pérez A, Ramos JA, Rodríguez-Pascual AF, Vilches-Blázquez LM

The IGN-E case: Integrating through a hidden ontology

The 13th International Symposium on Spatial Data Handling (SDH 2008)

June 23rd - 25th, 2008.

Montpellier, France.

Pages: 417-135

ISBN: 978-3-540-68565-4 


\title{
THE IGN-E CASE: INTEGRATING THROUGH A HIDDEN ONTOLOGY
}

\author{
Gómez-Pérez A, Ramos JA \\ Ontology Engineering Group - UPM, Spain \\ \{asun, jarg\}@fi.upm.es \\ Rodríguez-Pascual A, Vilches-Blázquez LM \\ National Geographic Institute of Spain (IGN-E), Spain \\ \{afrodriguez, Imvilches\}@fomento.es
}

\begin{abstract}
National Geographic Institute of Spain (IGN-E) wanted to integrate its main information sources for building a common vocabulary reference and thus to manage the huge amount of information it held. The main problem of this integration is the great heterogeneity of data sources. The Ontology Engineering Group (OEG) is working with IGN-E to attain this objective in two phases: first, by creating automatically an ontology using the semantics of catalogues sections, and second, by discovering mappings automatically that can relate ontology concepts to database instances. So, these mappings are the instruments to break the syntactic, semantic and granularity heterogeneity gap. We have developed software for building a first ontology version and for discovering automatically mappings using techniques that take into account all types of heterogeneity. The ontology contains a set of extra-attributes which are identified in the building process. The ontology, called PhenomenOntology, will be reviewed by domain experts of IGN-E. The automatic mapping discovery will be also used for discovering new knowledge that will be added to the ontology. For increasing the usability and giving independence to different parts, the processes of each phase will be designed automatically and as upgradeable as possible.
\end{abstract}

Keywords: ontology creation, geographic information, feature catalogues, mapping discovery, ontology-database mapping, heterogeneity

\section{INTRODUCTION}

National Geographic Institute of Spain (IGN-E) wanted to integrate its information sources for building a common vocabulary reference and thus to manage the huge amount of information it held. The main reason was to offer a unified national vocabulary to different Geographical Information (GI) producers, which have different interest, necessities and work scale (national - regional - local).

IGN-E has four main databases that correspond to different scales: Conciso Gazetteer (NC) $(1: 1,000,000)$, National Geographic Gazetteer (NGN) (1:50,000), Numerical Cartographic Database (BCN200) $(1: 200,000)$ and Numerical Cartographic Database (BCN25) $(1: 25,000)$. Each database has a different constant table to store represented features and their attributes. These databases are maintained separately and present great heterogeneity in different issues as we will show below. 
The active collaboration between IGN-E and OEG (Ontology Engineering Group) of UPM (Universidad Politécnica de Madrid) aims to create an integration framework for maintaining the current databases. This framework will be designed and, in the future, added to databases, and it should be built following the most automatic processes in order to solve as best as possible the heterogeneity problems that may arise.

This paper is organized as follows. Section 2 presents the history and characteristics of the current catalogues involved in this integration work. Section 3 describes the problems found and the approach proposed to solve them. Section 4 focuses on heterogeneity types and levels. Section 5 covers the steps followed to integrate the different feature catalogues. Section 6 shows in detail the automatic ontology creation. Section 7 deals with the automatic mapping discovery. Finally, section 8 provides some brief conclusions and discusses some future lines of work.

\section{EXISTING CATALOGUES}

The IGN-E has various databases and feature catalogues, but this work focuses on four main data sources: two Numerical Cartographic Database (BCN25 and BCN200) and two gazetteers (Concise Gazetteer and National Geographic Gazetteer).

With regard to the two Numerical Cartographic Databases, we can point out that they are considered as feature catalogues. This type of catalogue presents the abstraction of reality, represented in one or more sets of geographic data, as a defined classification of phenomena. It defines the feature type, its operations, attributes, and associations represented in geographic data. This type of catalogue is indispensable to turning data into usable information (ISO 19110). Next we provide some details of these data sources.

BCN25 was designed as a derived product from the National Topographic Map, which was created at a 1:25,000 scale (MTN25) in 1997, whereas MTN was created at a 1:50,000 scale (MTN50) in 1870. This long and hard project culminated at the end of the 1960s leaving behind great many changes due mainly to the continuous evolution that affected cartographic techniques during those years. From 1975 onwards, the updating of maps was carried out simultaneously with the production of a new series of maps at a1:25,000 scale (MTN25) with the aim of complementing MTN50 with some areas of special interest. However, in the 1980s these new series became a national coverage project. This Numerical Cartographic Database (BCN25) was built to obtain the 1:25,000 cartographic information that complies with the required data specifications exploited inside Geographic Information Systems (GIS) environments. Therefore, BCN25 contains essentially the same information than MTN25, though it has some additional geometric and topological properties, following a specific database oriented model and feature catalogue (Rodriguez, 2005). The figure below shows a small part of the BCN25 feature catalogue. 


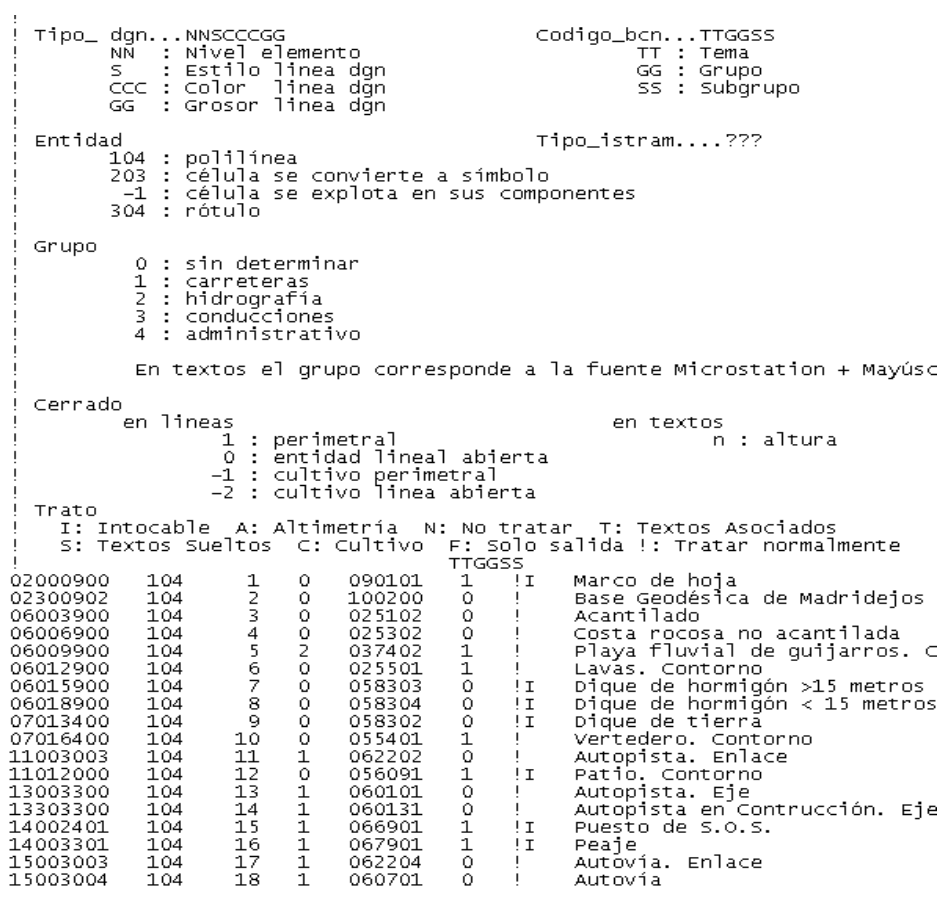

Figure 1. Source: BCN25

On the other hand, the first version of the Numerical Cartographic Database (BCN200) at a1:200,000 scale was started in 1985. This work was developed through analogical map digitalisation of provincial maps at this scale (Sevilla, 2006). Below, a part of the BCN200 feature catalogue is shown as an example of the layout that the catalogue presents.

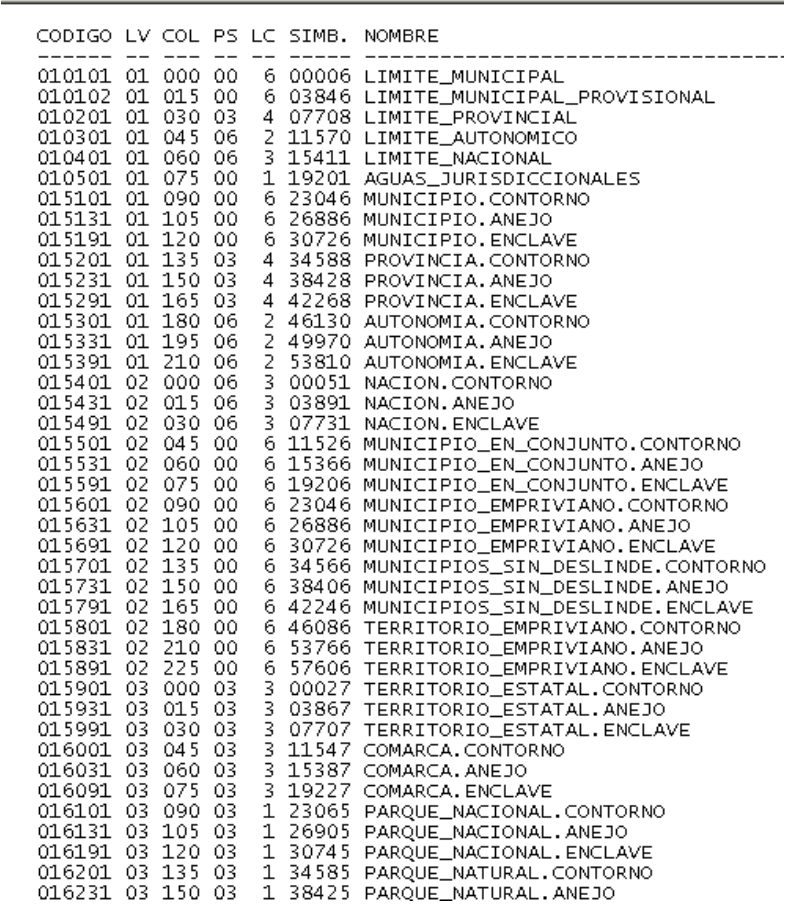

Figure 2. Source: BCN200

The information contained in the two Numerical Cartographic Databases is structured in eight different topics (Administrative boundaries, Relief, Hydrography, Vegetation, 
Buildings, Communications, Piping lines and Toponymy). Each topic is coded with three pairs of digits: two for its topic, two for its group (part of homogeneous information structured in topics) and two for its subgroup (a stretch of geographic feature belongs to a group). These numbers describe and classify different features regardless of its location and spatial dimension. The following text box shows an example of how this information has been structured.

\section{Topic 03: Hydrography}

Group: 01 constant watercourse

Subgroup: 01 Watercourse symbolized with one line

Figure 1 and 2 have other codes to symbolize graphical characteristics, which are associated to Computer Aided Design (CAD) Systems. These digits belong to the following attributes: level (LV), colour (COL), weight (PS) and style (LC).

The BCN25 feature catalogue has a peculiarity. This peculiarity appears as attributes named DGN_Type (“Tipo_dgn”), Entity (“Entidad”) or Group (“Grupo”), which represent an alternative way for structuring these geographical features. These attributes are shown in figure 1 and are subdivided into different categories. An odd case is the classification proposed by the "Group" attribute, since it represents a brief, but similar classification within this catalogue. It is subdivided into five topics (No specified, Roads, Hydrography, Piping lines and Administrative).

As regards gazetteers, a widespread definition of this concept comes from (ISO 19112). This standard defines a gazetteer as a directory of instances of a class or classes of features that contain some information regarding position. Next, some of the main characteristics of the IGN-E gazetteers are described.

The National Geographic Gazetteer (see figure 3), also called Georeferenced DataBase or NOMGEO, has 460,000 entries, which belong to different features in Spanish, Galician, Catalan, Basque and Aranes (official languages of Spain). This gazetteer has 14 items with Universal Transverse Mercator (UTM) and geographic coordinates. Moreover, the gazetteer is the information source of the Web Service of the Spanish Spatial Data Infrastructure (IDEE) ${ }^{1}$.

The Conciso Gazetteer (see Figure 3) is a basic corpus of standardized toponyms created by the Spanish Geographical Names Commission. The first version has 3667 toponyms. This gazetteer agrees with the United Nations Conferences Recommendations on Geographic Names Normalization. Furthermore, the gazetteer has 17 items, of which some are mandatory: Name, Name Language, Group, Feature Type, Province, Autonomous Region, Latitude, Longitude, Map and Name Source; and others, optional: Variant, Variant Language, Before, Before Language, Municipality, Variant Source and Observations. These items are in accordance with the Spanish Gazetteer Model $^{2}$. The Conciso Gazetteer has been created by the Spanish Geographical Names Commission. For further details, refer to (Nomenclátor Geográfico Conciso, 2006).

\footnotetext{
${ }^{1}$ http://www.idee.es

${ }^{2}$ http://www.idee.es/resources/recomendacionesCSG/MNEv1_2.pdf
} 


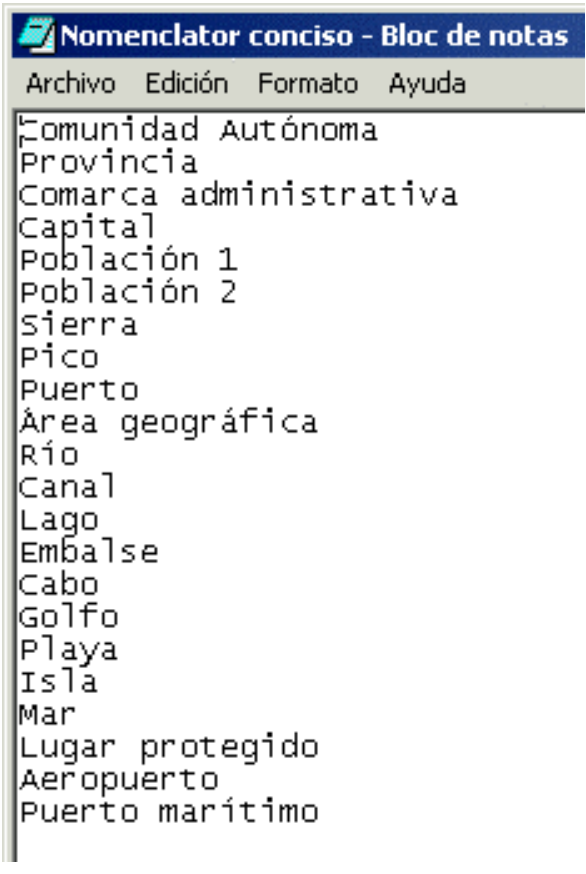

\section{Conciso Gazetteer}

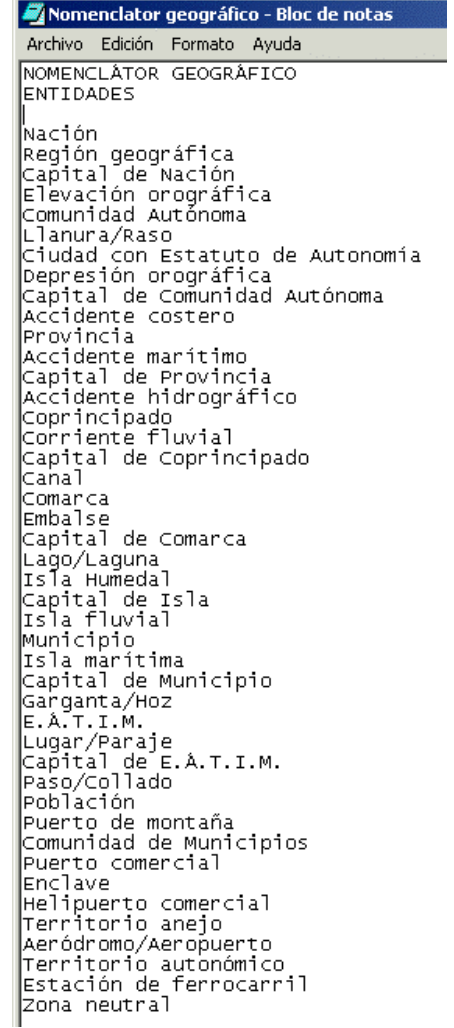

National Geograbhic Gazetteer

Figure 3. Feature Types of IGN-E Gazetteers

Regarding previous data sources, there is, in some cases, a mix between geographical and cartographic concepts. On the one hand, in IGN-E gazetteers, only geographic concepts such as Reservoir ("Embalse”), Province ("Provincia”) Plain ("Llanura”), etc. appear, whereas in the BCN feature catalogues we can only find concepts specific to the GI domain (as province, river or dam) and some of their geometrical characteristics (as outline, axis, symbolized by one line and so on). This peculiarity will not have any influence on the development of our work.

\section{PROBLEMS AND THE PROPOSED APPROACH}

From a general viewpoint, GI is increasingly captured, managed and updated with variable levels of granularity, quality and structure by different cartographic agencies. In practice, this approach causes the building up of multiple sets of spatial databases with a great heterogeneity of feature catalogues and data models. That means a coexistence of a great variety of sources with different information, structure and semantics without a general harmonization framework. On the other hand, this heterogeneity combined with the sharing needs of miscellaneous users and information overlaps from different sources, causes several and important problems when linking similar features, to search, retrieve and exploit GI data (Vilches et al., 2007a).

From a narrow viewpoint, the most important concept for GI is the feature since the Open GeoSpatial Consortium (OGC) (OGC, 2003) has declared that a geographic feature is the starting point for modelling geospatial information. For that reason, the basic unit of GI within most models is the "feature", an abstraction of a real world 
phenomenon associated with a location relative to the Earth, about which data are collected, maintained and disseminated (ISO 19110). Features can include representations of a wide range of phenomena that can be located in time and space such as buildings, towns and villages or a geometric network, geo-referenced image, pixel or thematic layer. This means that, traditionally, a feature encapsulates in one entity all that a given domain considers about a single geographic phenomenon (Greenwood et al., 2003). From this point of view, we can observe that the heterogeneity associated to the feature term grows more because of the interests and necessities of different GI producers.

From an ontological perspective, no ontology has compiled the characteristics and peculiarities of Spain's geographic features. Up to now, there is only a hydrographical feature ontology of these characteristics, called hydrOntology (Vilches et al., 2007b). On the other hand, the use of standardized vocabularies, such as CORINE Land Cover ${ }^{3}$, EuroGlobalMap ${ }^{4}$ or EuroRegionalMap ${ }^{5}$ involves an oversimplification of the existing complex reality because each GI producer (both national and local) has different feature catalogues (following their self-interests), which provokes that the overlaps between features are, quite often, not totally evident.

Taking into account these reasons, we decided to design an integration framework without having to reuse the information technology (standards, ontologies, feature catalogues, etc.) available. Figure 4 shows the approach proposed for this integration, which implies using an ontology of features while keeping the current databases.

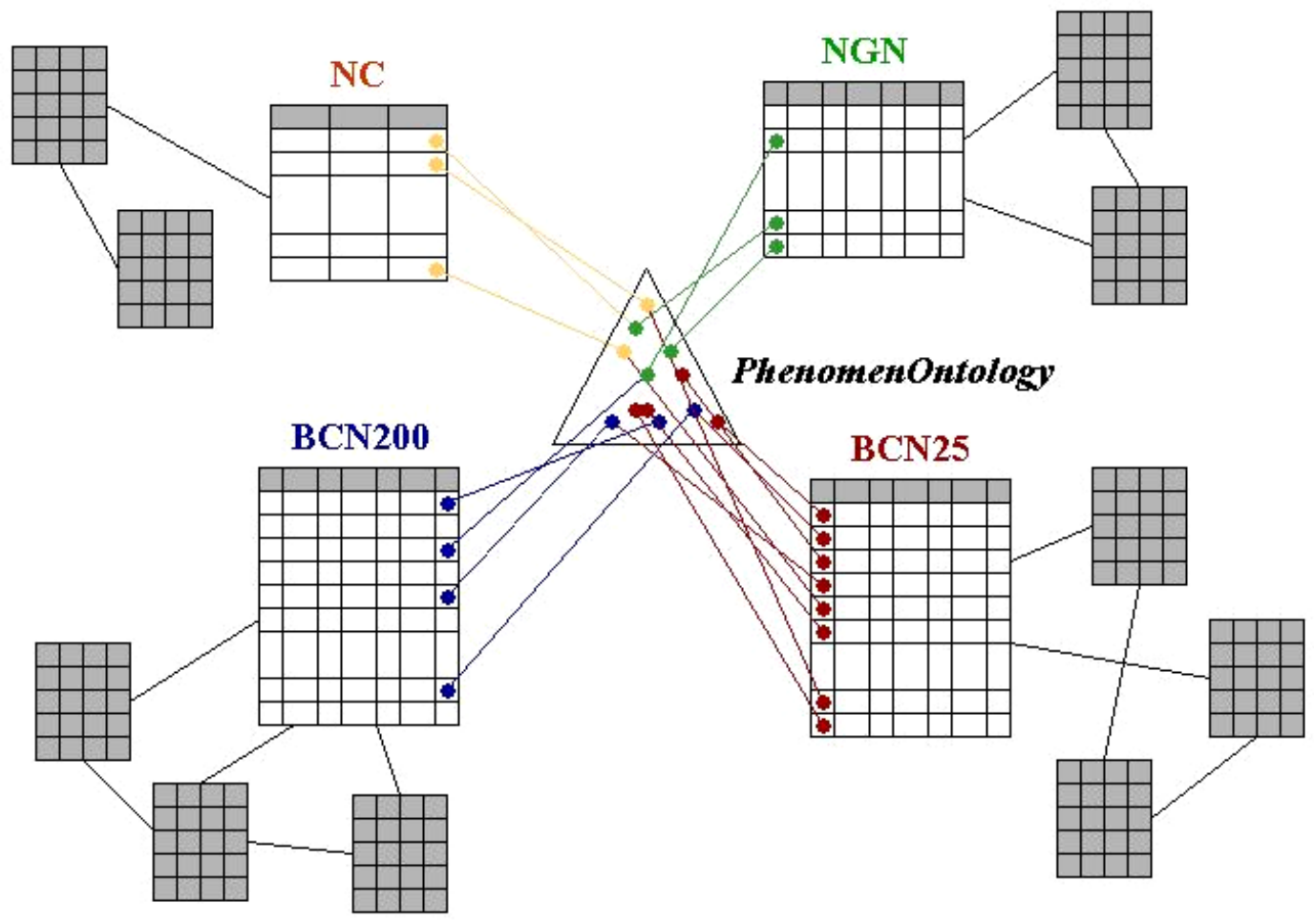

Figure 4: Proposed approach

\footnotetext{
${ }^{3}$ http://dataservice.eea.europa.eu/dataservice/metadetails.asp?id=950

${ }^{4} \mathrm{http}: / /$ www.eurogeographics.org/eng/03_projects_EGM_overview.asp

${ }^{5} \mathrm{http} / / /$ www.eurogeographics.org/eng/03_projects_euroregionalmap.asp
} 
As can be seen, an ontology (called PhenomenOntology) conceptualises features, and their concepts will be mapped to their corresponding phenomenon row in each database.

For integrating GI by means of ontologies, some authors propose a multi-ontology system (Stuckenschmidt et al., 1999) (Hakimpour et al., 2001) (Fonseca et al., 2002). In such approach each organization integrates its information sources using a local ontology; other ontology integrates all the organization ontologies of the system. According to this approach, our integration framework will be an organization inside a multi-ontology system.

Following the classification of ontologies for the geographic world provided by (Fonseca et al., 2002), in our framework we will build a Phenomenological Domain Ontology (PDO) that we will name PhenomenOntology.

\section{HETEROGENEITY}

In the geographical information domain, any differences in data sources, disciplines, tools and repositories can cause heterogeneity (Alonso et al., 1994). Next, we describe the different approaches that tackle heterogeneity problems.

In (Bishr, 1998), three different heterogeneity types (semantic, schematic and syntactic) are distinguished. First, semantic heterogeneity is usually the source of most of data sharing problems. This occurs because of the variation of models of the different disciplines and necessities, though geographical features are likely to share a common interest. Heterogeneity is subdivided into cognitive heterogeneity and naming heterogeneity. Cognitive heterogeneity is frequent when there is not a common base of definitions for the common features of different catalogues or databases, whereas naming heterogeneity is due to semantically alike features that might be named differently. For instance, watercourse and river are two names describing the same thing. On the other hand, in schematic heterogeneity the classification and hierarchical structure of the geographical feature could vary within or across disciplines. Finally, syntactic heterogeneity is divided in two types, one is related to the logical data model and its underlying DBMS (DataBase Management System), e.g., relational and object oriented, while the other is related to the representation of the spatial objects in the database.

Another approach to classify heterogeneity problems has been developed by (Hakimpour, 2003). This proposal puts forwards other classification, which presents similar aspects of heterogeneity to the previous one

- Heterogeneity in the conceptual modeling: A geographical feature can be represented in one system as an object class, and in other, as a relation.

- Heterogeneity in the spatial modeling: This feature type could be represented by polygons (or a segment of pixels) in one system, while being represented by lines in the second system.

- Structure or schema heterogeneity: In this heterogeneity type it is possible that different systems hold the same name for a same feature, but different attributes or formats. Therefore, the information of each system is different.

- Semantic heterogeneity: One system may adopt a viewpoint about a feature, while the other may adopt a different one. Moreover, it is usual to find different definitions of a same feature. 
In these classifications, big differences of granularity in a same domain do not appear as a heterogeneity problem; however, heterogeneity problems are presented in our catalogues because the conceptual overlap is complete, so the number of concepts that describe the geographical domain range from 22 to over 400 . This gap represents other type of heterogeneity which will be taken in account.

For solving the different problems caused by heterogeneity we will have to use mapping techniques. In our integrated system, mappings are the components which relate heterogeneous elements. So, mappings have to solve the heterogenity gap between ontology and catalogues.

\section{FROM PROPOSAL TO REALITY}

We have carried out the integration task in two phases: first, the building of PhenomenOntology, and then the mapping of the catalogues with PhenomenOntology.

To build the ontology, the members agreed to generate automatically an ontology that could cover as best as possible different feature domains (Administrative boundaries, Vegetations, Buildings, etc). For that, the domain experts decided to use the BCN25 catalogue, which is the most detailed one, for extracting the information and then with that information creating the ontology automatically. Once the ontology is created, the domain experts will have to review and modify it to cover all features presented in other catalogues.

To build mappings, OEG is creating a framework which permits adding new techniques for mapping discovery between our ontology and a database table. The discovery has to be automatic and the resultant alignments will be reviewed by domain experts. Here, we present how to include within the mapping discovery the automatic recognition of new knowledge for learning.

The process above described is carried out in two phases and to enhance usability such phases are designed as automatic sub-processes:1) For creating ontologies automatically, the configurable application, already built, permits experts to generate ontologies quickly and to evaluate the most appropriate taxonomic building criteria. 2) For mapping discovery, the automatic feature will permit to enlarge the system with other catalogues in the near future.

\section{AUTOMATIC ONTOLOGY CREATION}

\subsection{Scales and coverage}

As we have mentioned above, each of the IGN-E catalogues corresponds to a different scale. Therefore, the number of features is inversely proportional to the scale, because the detail permits distinguishing more specialized geographical features. Then, we use a $1: 25,000$ scale catalogue to generate automatically an ontology using an ad hoc application. This application extracts from instances of a feature catalogue the concepts of PhenomenOntology. 
Theoretically, the list of the smaller scale catalogue includes all the features of the larger scale, but this is not truth since a small number of features do not appear in the list of the smallest scale features. Examples of these features are: "cordillera" (mountain chain) or "península" (peninsula), these features cannot be drawn in a map following the smallest scale and these names only appear in a 1:1,000,000 scale catalogues. These special features or terms will be considered during the debugging phase carried out by experts of the domain, though we pretend to identify these terms in the mapping phase.

\subsection{Criteria for taxonomy creation}

The software developed for the automatic creation of an ontology permits selecting the criteria for taxonomy creation and its order. These criteria are based on the information contained in each row of the BCN25 feature catalogue table. First, the column codigo (code) stores codified information about a three-level taxonomy. Therefore, there are three separate criteria from which to extract a superclass in the taxonomy creation process: the first pair, the second pair, and the third pair of code digits. Then, the application permits extracting a super class for each different value of the chosen pair of digits. For increasing the versatility, the application permits selecting one of these pairs of digits, making possible to extract the taxonomy in different fashions: the first level of extraction attending the first pair of digits, the second level of extraction attending the third pair; or attending firstly the second pair and secondly the first pair; or any combination of one, two or three levels.

Three levels can result insufficient for a taxonomy with more than eight hundred leaves. So, we have added another criterion for creating automatically a taxonomy level: common lexical parts. At the beginning of the features, the application can identify common substrings of feature names and then create a common superclass of those concepts whose names begin with an identified substring. See an example of these criteria: we start with concepts “Autovía”, “Autopista 2 carriles”, “Autopista 3 carriles puente" and "Autopista 3 carriles túnel" that are siblings; when this criterion is applied, it produces a superclass called "Autopista" (sibling of "Autovía") which has as subclasses "Autopista 2 carriles", "Autopista 3 carriles puente” and "Autopista 3 carriles túnel". Applying this criterion twice, the software produces a taxonomy that has "Autovía" and "Autopista" as siblings; then "Autopista 2 carriles" and "Autopista 3 carriles" are siblings and sons of "Autopista"; and "Autopista 3 carriles puente" and “Autopista 3 carriles túnel” are siblings and sons of “Autopista 3 carriles”.

While testing the software, IGN-E and OEG noticed that the substring criterion is not useful because there is lexical heterogeneity in phenomenon names. Therefore, we created another substring criterion to solve the heterogeneity problems mentioned above, and as a result the substrings "Autovía”, "AUTOVIAS”, "Autovia." and "autovía-" are the same when we create a superclass.

According to the atributes of the BCN25 feature catalogue table, we created another criterion to extract superclasses (a new taxonomy level) for different values of grupo (group) that represents a top level classification of feature (as the first pair of code digits). 
There are a total of six criteria which can be ordered and combined as we wish, while a same criterion can, sometimes, be included in the criteria list. Figure 5 shows a schematic example of three criteria.

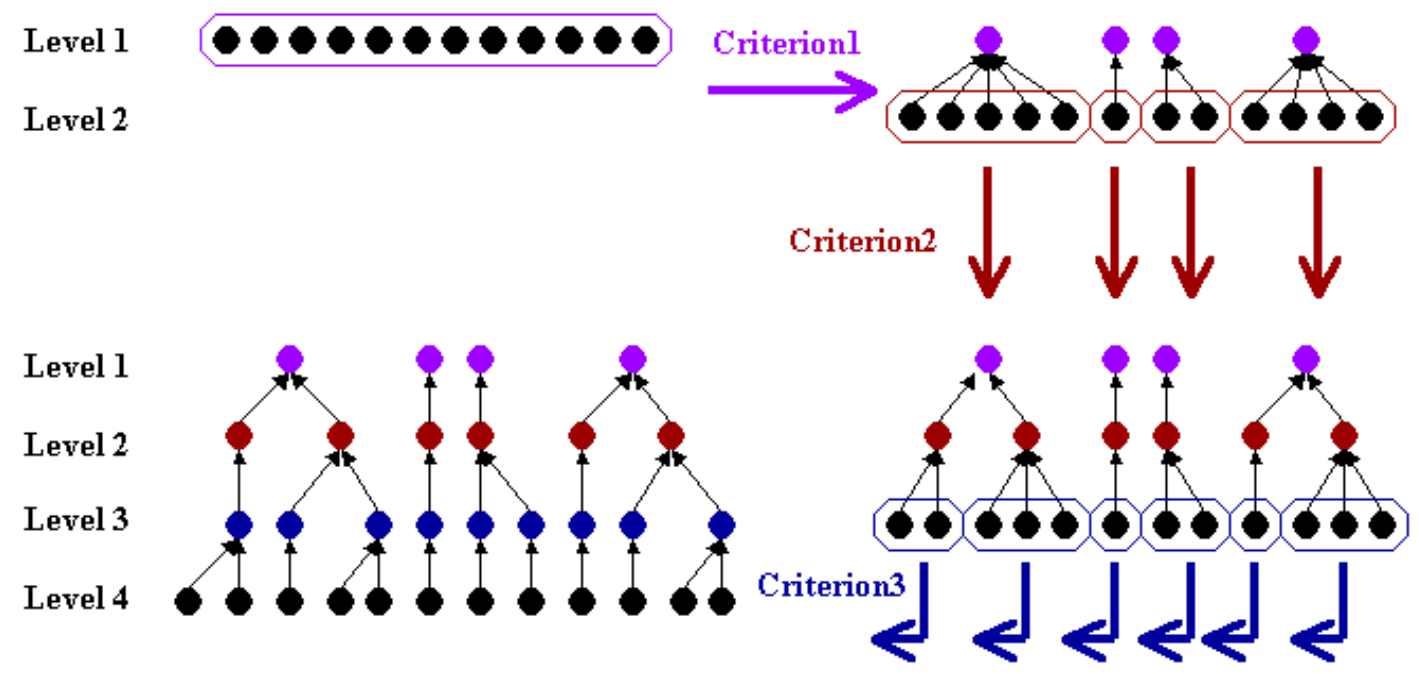

Figure 5: Example of taxonomic level creation

\subsection{Attributes by values}

We can find other type of information in feature names that can be quite interesting for ontology conceptualisation. Indeed, feature names in databases contain the values of conceptual attributes (called data properties in the description logics paradigm) that are not explicit in database models. For example, the state of a railway, which can be "en uso" (in use), “en construcción” (under construction), “abandonado” (abandoned) or "desmantelado" (dismantled). There is an opportunity for enrichment the conceptualization attending to these attribute values held in labels. We can upgrade the scanning values by inserting concept attributes with the values found during the reviewing of the concepts.

We had the opportunity of including within the ontology creation software an analyser of feature names. To do that, the software uses a file containing the attribute names and their values; the concepts of the ontology created can have more attributes than databases columns, taking these extra-attributes the values appearing in the name. For example, the concept "FFCC doble desmantelado" (double railway dismantled) will have two extra-attributes: "número de vías" (number of tracks) with value "doble" (double) and "estado" (state) with value "desmantelado" (dismantled). The number of extra-attributes or their values is open for this application.

\subsection{PhenomenOntology}

IGN-E used the application developed for generating criteria combination tests and chose an automatic generated ontology with three levels (two criteria), 686 concepts and 3,846 attributes, as can view in Figure 6. This ontology is stored in the WebODE platform (Arpírez et al., 2003). 


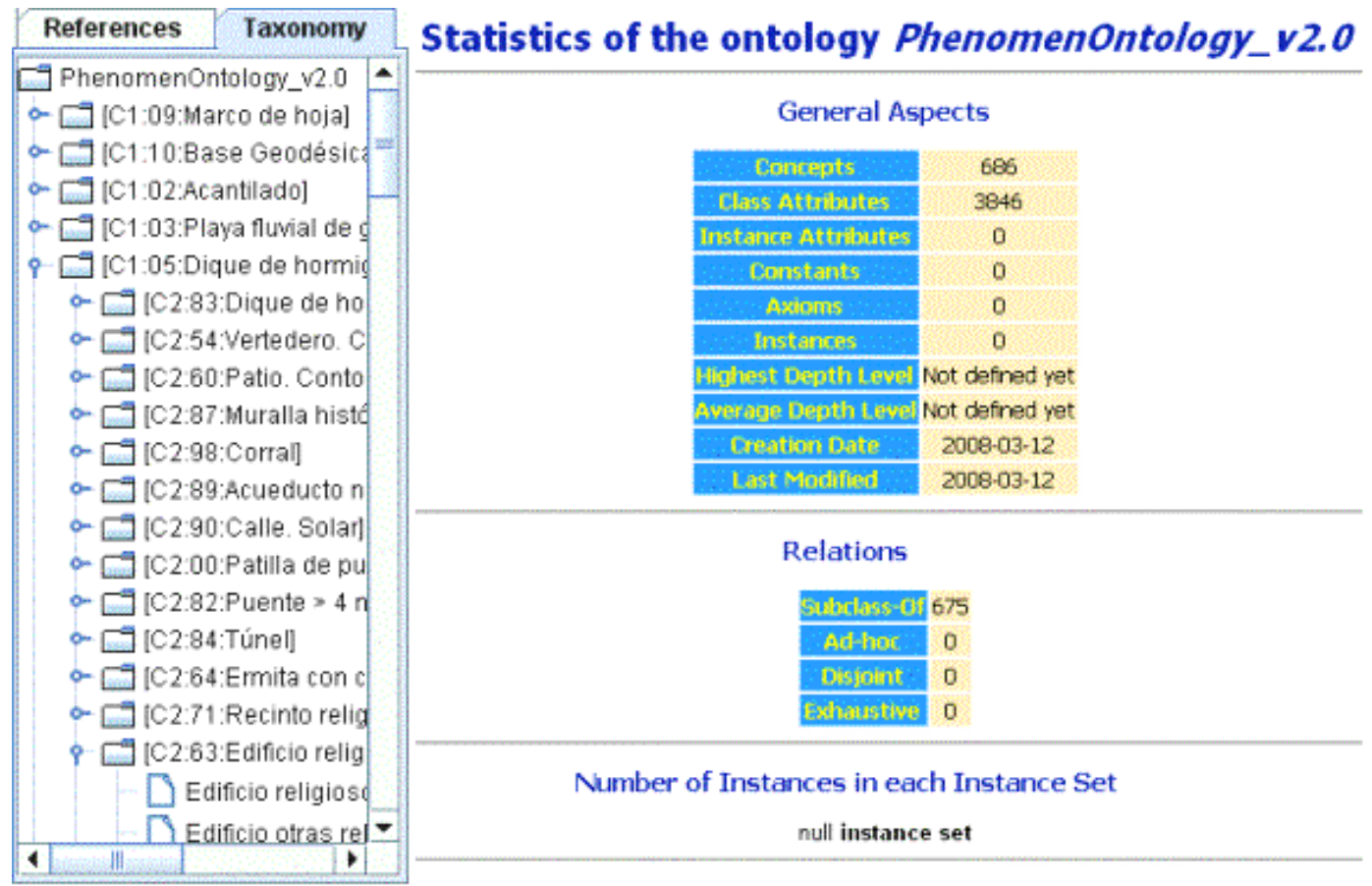

Figure 6. PhenomenOntology v2.0 and its statistics

This first version of the ontology is being refined by IGN-E experts using the WebODE Editor.

\section{AUTOMATIC MAPPING DISCOVERY}

In the approach here presented there are elements that relate feature stored in databases to concepts of PhenomenOntology. These elements are mappings.

The sets of mappings are classified into intensional and extensional; they are intensional when mappings relate elements of different set of instances, and they are extensional, when mappings relate elements of different conceptualizations. In our case, mappings relate instances of a model (rows of a table of a Relational Model) to concepts (elements of an ontology conceptualisation). We have not found similar cases in the literature and only a definition of mapping covers this type of relations. This definition is: "A mapping is a formal explicitation of a relation between elements, or set of elements, of different conceptualizations and/or instantiations.” (Gómez-Pérez et al., 2008).

Automatic mapping discovery is a traditional work area in which many algorithms and tools are developed; but due to our specific scenario, described above, we need to create new tools and algorithms, studying the existing reuse techniques (distance measures, matching terms, etc.).

We have identified several techniques to discover mappings following the analysis of feature catalogue. Below we present different analyses to review coverage of different heterogeneity types:

- Syntax analysis: String comparison. Before making string comparison, it would be necessary to regularize the string format (capitals, blank spaces, plurals, etc.). This kind of analysis solves, partially, semantic heterogeneity. Example: "autovia”, "Autovía.". 
- Syntax analysis: Regular expression. The inclusion of names into other names might represent hiperonymy/hiponymy information. This kind of analysis solves, partially, granularity heterogeneity. Example: “autovía”, “Autovía en construcción”.

- Semantic analysis: Hiperonymy. If it is possible to access linguistics resources where looking for hiperonymy information between names or part of names. This kind of analysis solves, partially, granularity heterogeneity. Example: "muro", "recinto amurallado".

- Semantic analysis: Synonymy. If it is possible to access to linguistics resources to look for synonymy information between names, acronyms or abbreviations. This kind of analysis solves, partially, semantic heterogeneity. Example: "muro", "pared exterior".

- Semantic analysis: Root. The comparison of roots of lemmas can provide information about synonymy. This kind of analysis solves, partially, semantic heterogeneity. Example: "muro”, “muralla”.

- Semantic analysis: Definitions. IGN-E provides mapping discovery with a set of definitions about features. With these definitions we will discover new synonymy and hyponymy relations. This kind of analysis solves, partially, semantic and granularity heterogeneity. For instance: "muro", "muralla: muro que rodea un recinto fortificado".

- Code analysis. Codes are identifiers and we use this code information (see code description in section 2) to identify synonymy relations. This kind of analysis solves, partially, semantic heterogeneity. For instance: "064401 Vías de estación de FFCC. Vía de servicio” (BCN25), “064401 FFCC.VIA_DE_SERVICIO” (BCN200).

- Structural analysis. As mentioned above, codes are composed of three pairs of digits with taxonomic information embedded. This taxonomic information can be used in catalogues with codes for scanning an ontology. This kind of analysis solves, partially, schematic heterogeneity. Example: "064204 FFCC en construcción en puente" (BCN25) implies 06 is transports, 42 is railways (a subclass of transport), and 04 is a subclass of railways.

\subsection{Knowledge discovery}

To facilitate the review and depuration of the automatic creation of the ontology, during the mapping discovery phase we have studied how to identify new knowledge while discovering mappings. Therefore, in the near future we will take in account two techniques:

- Code analysis. Codes are identifiers and we use code information (see code description in section 2) to identify synonymy relations between terms; then we reuse this semantics for mapping discovery. Example: "064301 FFCC abandonado o desmantelado” (BCN25), “064301 FFCC_FUERA_DE_SERVICIO”(BCN200). 
- Not identifying the relations with ontology concepts implies lacks of knowledge in the ontology added. Example: “068202 Radiofaro” (BCN200).

\section{CONCLUSIONS AND FUTURE WORK}

Our application for finding lexical heterogeneity in feature names has permitted IGN-E domain experts to evaluate its main data sources.

The automatic creation of ontologies and the easy combination of criteria have permitted us to have, very quickly, different first version ontologies for evaluation; thus expert time and efforts are saved. Other advantages of the application are the identification of extra-attributes by values in the feature names and the automatic storage in an ontology management platform such as WebODE, which permits debugging the ontology by experts easily.

The mapping discovery process is now being developed and we expect to obtain the first results in a few months. The process is being conscientiously developed with the aim of incorporating it to regional and international databases. In this mapping discovery, the application has to solve the heterogeneity problem in different levels so as to identify mappings automatically. However, the results of all the automatic processes must be reviewed by domain experts to get the most successful results.

As we mentioned above, the mapping discovery phase is not concluded yet. Therefore, this work is our priority for the near future.

Once the framework is finished, the collaboration partners will develop new techniques to discover mappings, which will improve this efficiency and will cover other type of relations

When the final phase of the mapping discovery framework is finished and the new techniques are added, then the integration system will be enlarged automatically with the incorporation of other catalogues (regional and local scale), extending the integration to other GI producers.

\section{ACKNOWLEDGEMENTS}

This work has been partially supported by the National Project "GeoBuddies" (TSI2007-65677C02) and the bilateral collaboration UPM-IGN 2007-2008. We are also grateful to Rosario Plaza for her help in checking and reviewing the grammar and spelling of the paper and improving clarity and style.

\section{REFERENCES}

Alonso G, Abbadi AE (1994) “Cooperative modelling in applied geographic research”. International Journal of Intelligent and Cooperative Information Systems, 3(1): 83-102

Arpírez JC, Corcho O, Fernández-López M, Gómez-Pérez A (2003) “WebODE in a nutshell”. AI Magazine, 2003. 
Bishr Y (1998) "Overcoming the semantic and other barriers to GIS interoperability". International Journal of Geographical Information Science, 12(4): 299-314.

International Standard Organization (ISO) (2003) ISO 19112:2003 “Geographic Information - Spatial referencing by geographic identifiers”.

Fonseca FT, Egenhofer MJ, Davis CA, Câmara G (2002) "Semantic Granularity in Ontology-Driven Geographic Information Systems". Annals of Mathematics and Artificial Intelligence. Volume: 36, 2002. Issue: 1-2, p. 121 - 151.

Gómez-Pérez A, Ramos JA (2008) "Semantic mappings: out of ontology world limits". Intl. Workshop on Ontology Alignment and Visualization. March 4-7, 2008. Barcelona, Spain. Pages: 907-912.

Greenwood J, Hart G (2003) "Sharing Feature Based Geographic Information - A Data Model Perspective”. $7^{\text {th }}$ Int. Conference on GeoComputation. United Kingdom.

Hakimpour F (2003) "Using Ontologies to Resolve Semantic Heterogeneity for Integrating Spatial Database Schemata”. Ph.D. thesis, Zurich University. Switzerland, 2003.

Hakimpour F, Timpf S (2001) "Using Ontologies for Resolution of Semantic Heterogeneity in GIS”. 4th. AGILE Conference on Geographic Information Science, Brno, Czech Republic, 2001.

ISO 19110 (2005) “Geographic Information” - Methodology for feature cataloguing.

ISO 19112 (2003) “Geographic Information” - Spatial referencing by geographic identifiers.

Nomenclátor Geográfico Conciso de España, versión 1.0. "Presentación y Especificaciones”. Instituto Geográfico Nacional. Octubre 2006. http://www.idee.es/ApliVisio/ Nomenclator/NGCE.pdf

OGC (2003) “OpenGIS Reference Model”, Version 0.1.2, OGC Inc. Wayland, MA, USA.

Rodríguez Pascual AF, García Asensio L (2005) “A fully integrated information system to manage cartographic and geographic data at a 1:25,000 scale". XXII International Cartographic Conference (ICC2005). A Coruña, Spain. ISBN: 0-958-46093-0

Sevilla Sánchez, C, Rodríguez Pascual A.F., González Matesanz FJ, Blanco Ortega LM, Vilches-Blázquez LM (2006) "Un SIG corporativo en el IGN para la gestión integrada, publicación y análisis de datos geográficos”. In proceedings of XII Congreso Nacional de Tecnologías de la Información Geográfica. Camacho Olmedo, M.T.; Cañete Pérez, J.A.y Lara Valle, J.J. Ed. ISBN: 84-338-3944-6 Depósito Legal: GR-1855-2006

Stuckenschmidt H, Visser U, Schuster G, Vögele T (1999) “Ontologies for geographic information integration". Proceedings of Workshop Intelligent Methods in Environmental Protection: Special Aspects of Processing in Space and Time, 13. 
International Symposium of Computer Science for Environmental Protection, CSEP 1999, pp. 81-107.

Vilches-Blázquez LM, Rodríguez Pascual A F, Mezcua Rodríguez J, Bernabé Poveda MA, Corcho O (2007a) "An approach towards a harmonized framework for hydrographic features domain”. In Conference Proceedings of XXIII International Cartographic Conference. 4-10 August 2007. Moscow, Russia.

Vilches Blázquez LM, Bernabé Poveda MA, Suárez Figueroa MC, Gómez-Pérez A, Rodríguez Pascual A F (2007b) “Towntology \& hydrOntology: Relationship between Urban and Hydrographic Features in the Geographic Information Domain”. Ontologies for Urban Development Ed. Teller, J.; Roussey, C.; Lee, J. Springer-Verlag, 2007. ISBN: 978-3-540-71975-5. 